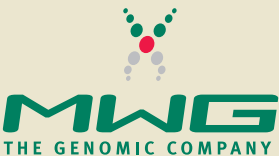

\title{
siRNA design induding secondary structure target site prediction
}

\author{
Gerrit Schramm \& Rebecca Ramey \\ When performing RNA interference (RNAi) experiments, the first critical challenge is the design of efficient \\ small interfering RNAs (siRNAs). MWG Biotech has created an online design tool allowing researchers to \\ analyze mRNA target sites.
}

\section{RNAi mechanism and history}

RNAi is the gene silencing process that results when the presence of double-stranded RNA induces cleavage of the mRNA. The double-stranded RNA is processed by an RNase III family enzyme Dicer, resulting in the generation of an siRNA, a 21-23nucleotide (nt) RNA duplex, composed of a 19-mer sequence with symmetric $2-3$-nt $3^{\prime}$ overhangs. The siRNA associates with cellular proteins to form the RNA-induced silencing complex (RISC). The siRNA antisense strand guides RISC to the target mRNA, resulting in endonucleolytic cleavage.

Important features in designing siRNA are specific target recognition and minimization of off-target effects. A set of rules for detecting 21-mer target sites was proposed through a systematic search on effective target sites ${ }^{1}$. A screen of 180 siRNAs on two genes resulted in a scoring system of siRNA quality with factors such as low $\mathrm{G}+\mathrm{C}$ content, a lack of internal repeats, an $\mathrm{A} / \mathrm{U}$-rich $5^{\prime}$ end and other features that enhance the silencing effect of siRNA ${ }^{2}$.

\section{Optimization methods}

Recently it was reported that 27 -mer duplexed nucleotides may offer a potency advantage over the more traditional 21-mer siRNA $^{3}$. It has been proposed that these longer double-stranded RNA molecules load more efficiently into the RISC complex because they undergo natural cleavage by Dicer. These 27-mers have also been shown to have the ability to silence mRNAs when 21-mer duplexes had previously failed.

Using bioinformatics software to reduce off-target effects has also become standard practice. Typically, a basic local alignment search tool (BLAST) search of organism-specific mRNA databases is used to identify potential off-targets.

Recent publications analyzing the relationship between local free energy and siRNA efficiency ${ }^{4,5}$ have demonstrated that mRNA target site secondary structure is of great importance for siRNA efficiency.

MWG Biotech, Anzinger Strasse 7a, 85560 Ebersberg, Germany. Correspondence should be addressed to G.S. (gschramm@mwgdna.com).

PUBLISHED ONLINE 21 J ULY 2005; DQ:10.1038/ NMETH780

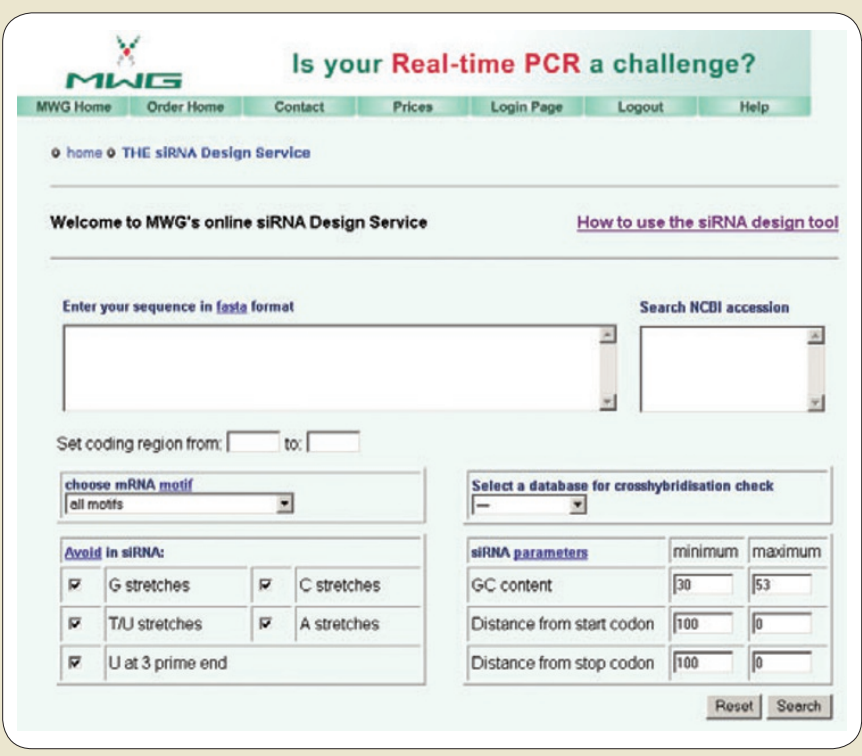

Figure 1 | MWG siRNA design tool interface.

\section{The MWG online siMAX siRNA design tool}

MWG's web-based siMAX siRNA design tool is free to use, has a full transparency and features a flexible design process, in which all design parameters can be customized, if desired (Fig. 1).

One or several genes can be input and the design algorithm then searches the DNA coding sequence for potential target sites, using all Tuschl motifs by default. In the next step (Fig. 2) siRNA is designed to target each of the corresponding target sites. The siRNA design algorithm avoids stretches with more than three identical nucleotides or a uracil at the $3^{\prime}$ end. Other factors influencing siRNA design are the $\mathrm{G}+\mathrm{C}$ content (default is between $30 \%$ and $53 \%$ ) and the distance of the siRNA from the start codon and the stop codon. The minimum distance from the start as well as from the stop codon is set at $100 \mathrm{nt}$ by default and is a critical parameter for design, especially with short coding sequences. It is recommended to vary these distances if the design algorithm fails initially at finding any siRNAs. 


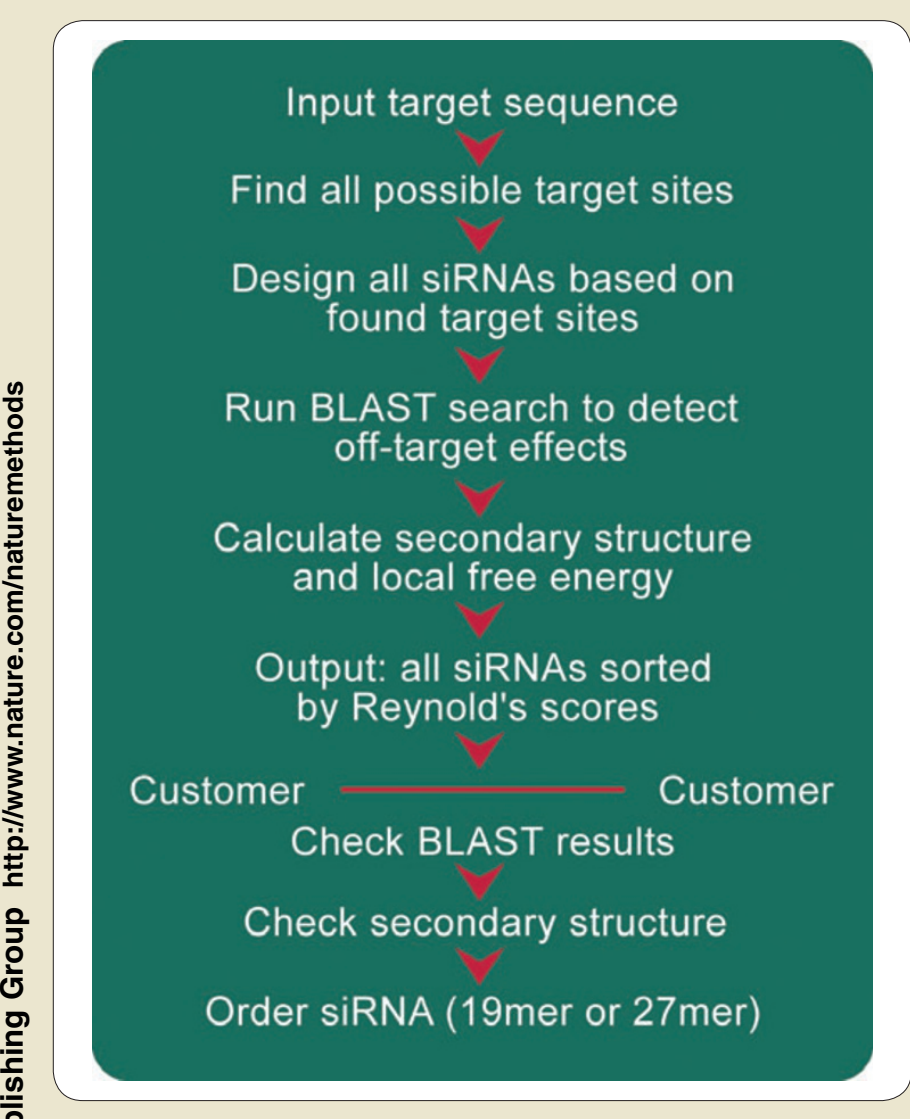

Figure 2 | Workflow of the MWG online siRNA design tool.

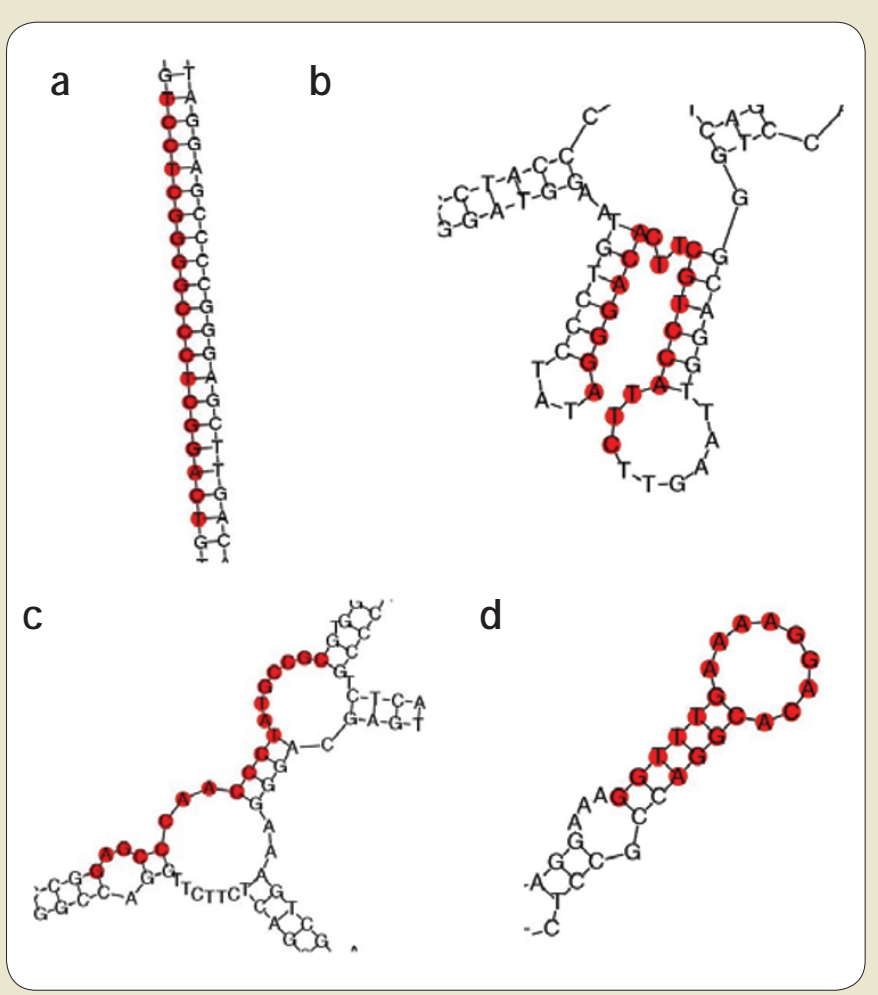

Figure 3 | Examples of siRNA target sites (red) on the corresponding mRNA secondary structure predicted using RNAfold. (a) Target site on a stack region. (b) Target site enclosed by two neighboring hairpin structures. (c) Target site on a region with three internal loops. (d) Target site on a single hairpin.
A BLAST search can be added which will detect possible offtarget effects and can be reviewed on a separate BLAST output page. For each siRNA, all BLAST hits are shown with direct links to the NCBI web page.

The secondary structure of the mRNA target site has an important role in siRNA efficiency. Thus, we have included a secondary structure view of the mRNA with the target site colored red, as shown in Figure 3, using the RNAfold program of the Vienna RNA package (http://www.tbi.univie.ac.at/ ivo/RNA/RNAfold. html). As an additional useful parameter, we calculate the local free energy at the target site. With these new options the user can quickly identify highly efficient siRNAs. The customers can select candidates visually, or can use the local free energy data and number of bound bases as quality values.

\section{Influence of the secondary structure}

It is not fully understood how the siRNA-mRNA target hybrid is formed. The importance of secondary structure, however, is supported by studies, which have shown that a target region incorporated in various hairpin structures (Fig. 3b) within the target region will dramatically reduce the potency of gene silencing by siRNA ${ }^{4}$. There appears to be a linear correlation between siRNA silencing of the target gene and the local free energy of the target region. A low negative local free energy, which correlates to an open region with many unpaired nucleotides (Fig. 3c) results in improved potency ${ }^{4}$.

Because of these recent studies, MWG Biotech updated its design tool to display the secondary structure of the
siRNA/mRNA target site. By knowing both the number of bound bases as well as the local free energy value, customers are able to identify more efficient siRNAs and also have the flexibility to decide which features they wish to avoid. The ability to visually check the target site secondary structure is new in commercial design tools and simplifies the selection of highly efficient siRNAs. In doing so, MWG has given the customer greater flexibility in designing their siRNA as additional papers and algorithms are published.

MWG Biotech provides three different scales of high-quality siRNA, either desalted or high performance liquid chromatography (HPLC)-purified. siRNA duplexes are available in both 21mer and 27-mer lengths. Being completely integrated into MWG Biotech's online ordering system, the design tool allows the user to directly order the siRNA with a simple mouse click.

Additional information is available on the company's website (http://www.mwg-biotech.com).

1. Elbashir, S.M. et al. Duplexes of 21-nucleotide RNAs mediate RNA interference in cultured mammalian cells. Nature 411, 494-498 (2001).

2. Reynolds, A. et al. Rational siRNA design for RNA interference. Nat. Biotechnol. 22, 326-330 (2004).

3. Kim, D.H. et al. Synthetic dsRNA Dicer substrates enhance RNAi potency and efficacy. Nat. Biotechnol. 23, 222-226 (2005).

4. Schubert, S., Grunweller, A., Erdmann, V.A., \& Kurreck, J. Local RNA target structure influences siRNA efficacy: systematic analysis of intentionally designed binding regions. J. Mol. Biol. 348, 883-893 (2005).

5. Heale, B.S., Soifer, H.S., Bowers, C., \& Rossi, J.J. siRNA target site secondary structure predictions using local stable substructures. Nudeic Acids Res. 33, e30 (2005). 